\title{
Skimager for the objective erythema estimation in atopic dogs
}

Cugmas, Blaž, Olivry, Thierry, Olivrī, Alla, Spīgulis, Jānis

Blaž Cugmas, Thierry Olivry, Alla Olivrī, Jānis Spīgulis, "Skimager for the objective erythema estimation in atopic dogs," Proc. SPIE 11211, Photonics in Dermatology and Plastic Surgery 2020, 1121110 (19 February 2020); doi: $10.1117 / 12.2544664$

SPIE. Event: SPIE BiOS, 2020, San Francisco, California, United States 


\title{
Skimager for the objective erythema estimation in atopic dogs
}

\author{
Blaž Cugmas*a , Thierry Olivry ${ }^{\mathrm{b}, \mathrm{c}}$, Alla Olivrī̄ ${ }^{\mathrm{d}}$, Jānis Spīgulis ${ }^{\mathrm{a}}$ \\ ${ }^{a}$ Biophotonics laboratory, Institute of Atomic Physics and Spectroscopy, University of Latvia, \\ 19 Rainis Blvd., Rīga, LV-1586, Latvia, EU; \\ ${ }^{b}$ Department of Clinical Sciences, College of Veterinary Medicine, NC State University, \\ 1060 William Moore Dr., Raleigh, NC, 27607 USA; \\ ${ }^{c}$ Comparative Medicine Institute, NC State University, Raleigh, NC, 27606 USA; \\ ${ }^{d}$ Veterinary Dermatology Riga, Pet City, 31 Pulkveža Brieža St., Rīga, LV-1010, Latvia, EU
}

\begin{abstract}
In this study, the severity of canine skin erythema was assessed objectively for the first time. Atopic dermatitis (AD) is a common canine inflammatory and pruritic skin disease associated with an allergic reaction to exogenous allergens. The monitoring of skin erythema over time with lesion severity scales like the CADESI-4 is an essential diagnostic and research tool, especially for clinical trials. Currently, the erythema assessment is subjective due to visual estimation. In our study, we calculated the erythema index (EI) in 14 atopic dogs based on the analysis of multispectral skin images taken with the Skimager device. The relationship between the EI and a visual erythema estimation was modeled by linear regression with the first-order polynomial. The coefficient of determination (r squared) reached 0.81. Based on such high correlation, we conclude that optical measurements could replace the visual estimation of erythema in atopic dogs and, thus, improve the validity of skin lesion severity scales in dogs.
\end{abstract}

Keywords: atopic dermatitis, erythema severity, erythema index, multispectral imaging, Skimager, dogs, disease severity scales, CADESI-4

\section{INTRODUCTION}

Atopic dermatitis $(\mathrm{AD})$ is a common canine chronic inflammatory skin disease, related to allergy ${ }^{1}$. The allergens like house dust mites, grass, and mold spores trigger the allergic reaction, which manifests as often-intense pruritus and skin lesions. Skin lesions (Fig. 1) can be primary (e.g., erythema), secondary due to chronic inflammation (e.g., lichenification and hyperpigmentation), or related to scratching (e.g., excoriations). The AD requires lifelong management, including the routine administration of antipruritic and anti-inflammatory drugs (given systemically or topically), immunotherapy, and good hygiene of the coat and skin. The treatment response in atopic dogs is highly individual. Therefore, the precise monitoring of clinical signs changes is crucial to modify or continue the anti-allergic therapy.

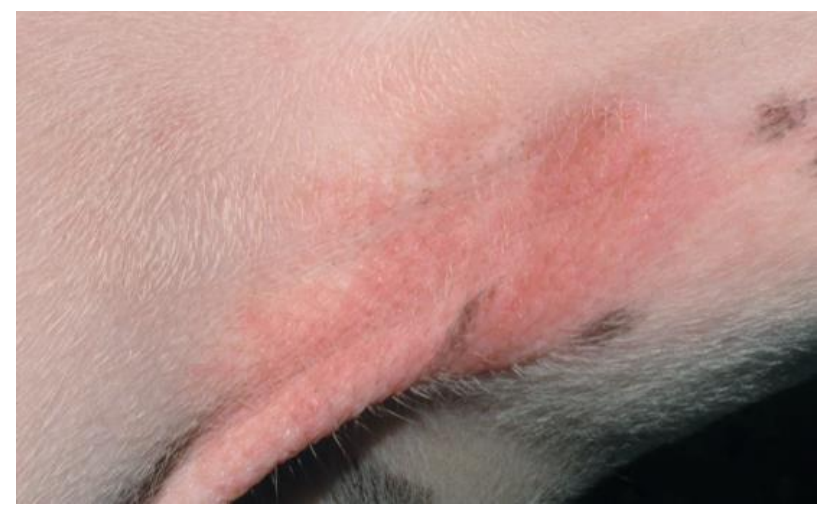

Figure 1. Skin from a Dalmatian dog with moderate erythema in the axillar region.

*blaz.cugmas@lu.lv; phone +371 67228249; http://www.asi.lv/

Photonics in Dermatology and Plastic Surgery 2020, edited by Bernard Choi,

Haishan Zeng, Proc. of SPIE Vol. 11211, 1121110 - (C) 2020 SPIE

CCC code: $1605-7422 / 20 / \$ 21 \cdot$ doi: $10.1117 / 12.2544664$ 
Various disease severity scales have been developed to monitor changes in pruritus and skin lesions. Dermatologists track the severity of itch with a pruritus visual analog scale (PVAS) ${ }^{2}$, ranging between 0 (no itch) and 10 (almost continuous itch). For monitoring skin lesions in atopic dogs, we use one of two major scales; the fourth iteration of the Canine Atopic Dermatitis Extent and Severity Index 4 (CADESI-4) ${ }^{3}$ or the Canine Atopic Dermatitis Lesion Index $(\mathrm{CADLI})^{4}$. The CADESI-4 requires 60 independent evaluations of three lesion types on 20 skin sites with the scores between 0 and 3, while the CADLI includes ten separate skin lesion estimations in five typically-affected regions with the scores between 0 and 5 .

Besides lichenification (due to chronic inflammation) and excoriation-alopecia (due to intense pruritus), erythema is a primary AD-related lesion that represents acute inflammation ${ }^{3,5}$. Therefore, both scales (CADESI-4 and CADLI) depend heavily on erythema estimation, especially when grading acute flares of the disease. However, making 20 (CADESI-4) or five (CADLI) erythema assessments can be time-consuming. Furthermore, visual assessments are highly subjective and impacted by factors like ambient illumination. Finally, both scales are discrete with a few possible score values (4 or 6 in CADESI-4 or CADLI, respectively), limiting their feasibility in the clinics and research ${ }^{6}$.

In human dermatology, erythema is assessed objectively with the help of commercial spectroscopic devices (e.g., Mexameter $\left.{ }^{\circledR}\right)$ or digital photography ${ }^{7}$. These approaches are typically based on an erythema index (EI), which is a ratio between spectral or image RGB components ${ }^{8}$. In dogs, $a^{*}$ values (CIELAB color space) estimated from colorimetry and RGB images have been shown to exhibit a limited capability to differentiate between various degrees of erythema severity ${ }^{9}$.

In our study, we tested a multispectral imaging device, Skimager, for objective erythema estimation in atopic dogs. First, we proposed a continuous (analog) scale, which allowed the simple matching of red shades with the severity of skin erythema. Secondly, we calculated four different types of EIs from Skimager's multispectral (blue, green, and red) skin measurements. Finally, calculated EI values were compared to the visual erythema scores based on the continuous scale and CADESI-4. We believe that objective erythema estimation could increase the validity of skin lesion severity scales in dogs.

\section{METHODS AND MATERIALS}

\subsection{Animals and measurements}

We selected 14 purebred or crossbred client-owned dogs of various ages and sizes with the final or the differential diagnosis of atopic dermatitis (AD) presented at our clinic.

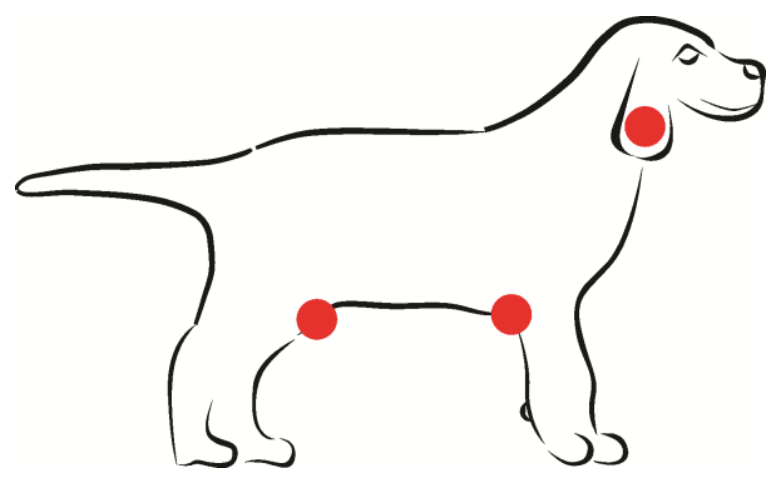

Figure 2. Erythema severity was estimated at three easy-accessible body sites: in the inguinal, axillar, and auricular region.

We studied erythema at three easy-accessible body sites (Fig. 2), which are commonly associated with AD (i.e., inguinal, axillar, and auricular regions). First, the dermatologist estimated the severity of erythema according to two scales; an ad hoc newly-created continuous scale and the CADESI-4 (Fig. 3). Secondly, multispectral images were taken by the Skimager. The device camera was held $5.0 \mathrm{~cm}$ away from the skin, resulting in $7.1 \mathrm{~cm}^{2}$ of the circular sampling area. 
All visual or multispectral estimations were done only on body sites without any additional lesions such as lichenification, excoriation, and hyperpigmentation. Additionally, if a body site included skin folds or a lot of hair, multispectral assessments were not performed.

The work was approved by the Latvian Food and Veterinary Service.

\section{No erythema}

\section{The most}

(a)

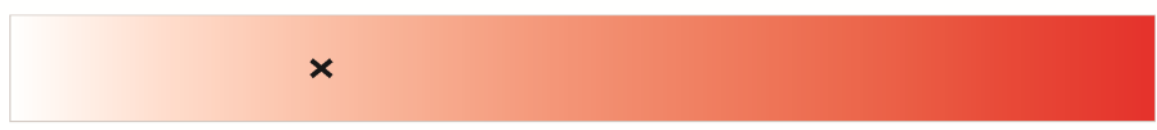

(b)

\begin{tabular}{|c|c|c|c|}
\hline $\mathbf{0}$ & $\mathbf{1}$ & $\mathbf{2}$ & $\mathbf{3}$ \\
\hline none & mild & moderate & severe \\
\hline
\end{tabular}

Figure 3. The severity of erythema was estimated according to (a) the continuous scale (manual valuation was transformed to a percentage) and (b) the established CADESI-4 scale with four steps between 0 (no erythema) and 3 (severe erythema). The example of an erythema estimation on both scales is shown as a cross (a) and a circle (b).

\subsection{Scales}

For visual erythema estimation, two scales were applied (Fig.3). First, a continuous (analog) scale included a red strip with the palette of red shades (from pale to intense red). The clinician marked a spot, corresponding to the severity of skin erythema. Later, the valuation was converted linearly to the percentage (no erythema $-0 \%$, the most severe erythema $-100 \%)$. The second scale was based on the CADESI- $4^{3}$, where a clinician rated the erythema as none $(0)$, mild (1), moderate (2), or severe (3).

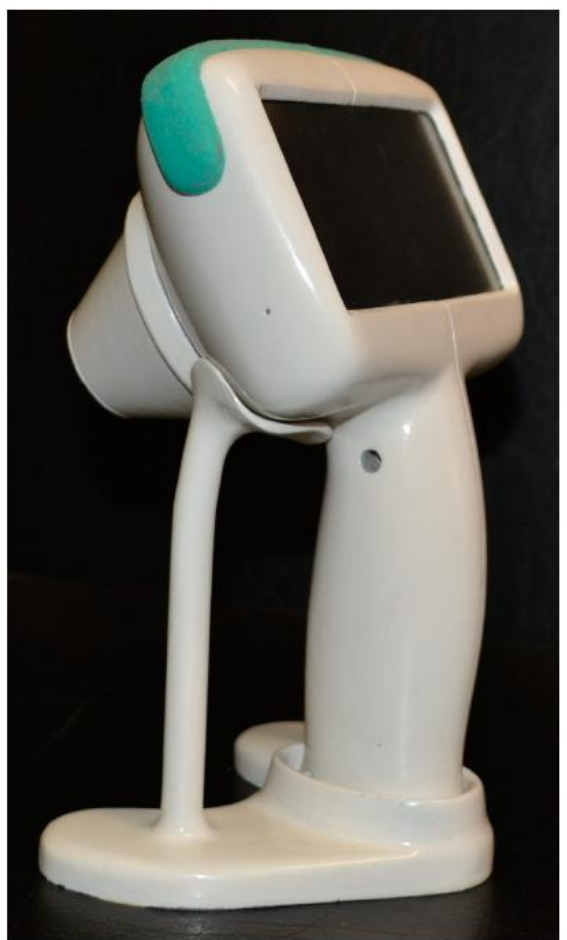

Figure 4. Skimager is a device for handy clinical measurements of skin properties ${ }^{12}$. 


\subsection{Skimager}

We performed multispectral measurements with the Skimager (Fig. 4), which is a device for multimodal skin imaging ${ }^{10}$. Skimager, with its affordable optical components, aims at a widespread clinical use providing clinically relevant skin information based on easy and handy measurements. The device acquires four consecutive data sets: (i) RGB image under white polarized LED (peak at $540 \mathrm{~nm}$ ); (ii) four spectral images based on narrowband LEDs with the peaks at $365 \mathrm{~nm}$ (blue), $450 \mathrm{~nm}$ (green), $660 \mathrm{~nm}$ (red), and $940 \mathrm{~nm}$ (IR); (iii) video under green LED illumination; and (iv) autofluorescence video or image under UV irradiation. From the acquired images and video, the information on skin chromophores, blood perfusion, and fluorophores can be obtained and displayed on the 4.3-inch touchscreen. Skimager includes a 3 Mpix RGB CMOS sensor with 3.2-micron pixel size (MT9T031, Micron Technology, Boise, USA), and a Tegra 2 T20 module (Nvidia, Santa Clara, USA) with a dual-core Cortex-A9 processor (ARM Inc., San Jose, USA). The Li-ion battery guarantees up to 100 full measurement cycles. Skimager was applied mostly on skin tumors ${ }^{11}$.

\subsection{Erythema index (EI)}

First, the multispectral images were normalized against the Classic ColorChecker's gray "neutral $8(.23 *)$ " patch (XRite, Grand Rapids USA). We used three manual approaches for sampling the acquired data (Fig. 5). The first method (PT) included 60 pixels, which were selected to represent an erythematous skin without pale spots and hair. Secondly, we selected pixels in two small squares $(S Q 2)$. Finally, only one large square was chosen (SQ1). Typical blue $(B)$, green $(G)$, and red $(R)$ values were calculated as an average from all the selected pixels. Finally, different EIs ${ }^{8}$ were estimated as:

$$
\begin{gathered}
\mathrm{EI}_{\mathrm{RG}}=\frac{R}{G}, \\
\mathrm{EI}_{\mathrm{GR}}=\frac{G-R}{G+R}, \\
\mathrm{EI}_{\mathrm{BRG}}=\frac{\sqrt{B \cdot R}}{G}, \\
\mathrm{EI}_{\mathrm{BG}}=\frac{B}{G^{2}} .
\end{gathered}
$$

We analyzed the relationship between variables (scales and EIs) with Spearman correlation and linear regression in the Matlab environment (R2017b, MathWorks, Natick, USA).

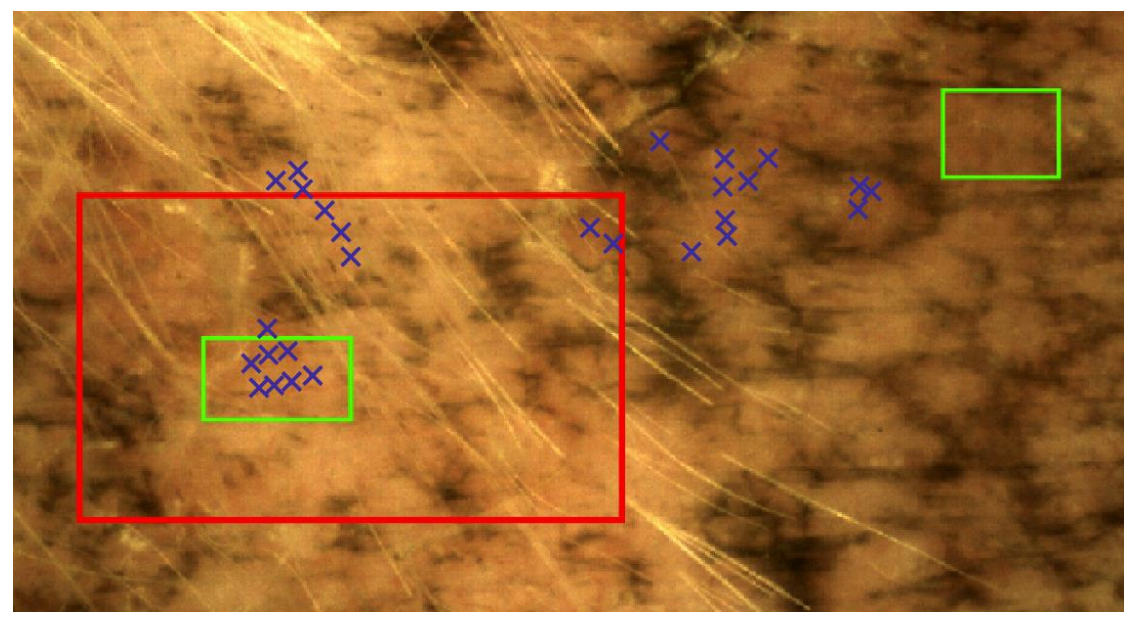

Figure 5. The sampling approach. Erythema index (EI) calculation was based on 60 pixels (blue, PT), and all the pixels in two small (green, SQ2) or one big square (red, SQ1). For the sake of simplicity, only a few selected pixels are shown for the PT approach. 


\section{RESULTS AND DISCUSSION}

We examined 26 body sites in 14 dogs for erythema severity according to the two selected scales (Fig. 3). We found a strong correlation between the proposed continuous and established CADESI-4 scale with the Spearman coefficient of 0.92 (Fig. 6). There was only a slight overlapping between two CADESI-4 groups of scores 0 (analog value $=6.7$ ) and 1 (analog value $=4.9)$.

The analog scale proved very convenient for erythema estimations in dogs with borderline CADESI- 4 scores. With the latter, a clinician has to select only one integer since no interim steps are allowed, causing a bias. On the other hand, the proposed scale allowed for an entirely free erythema severity assessment, based on color matching between the skin and the red strip. Since our study did not include any patients with severe erythema (CADESI-4 score of 3), we cannot judge if the selection of minimal and maximal red intensity and color distribution were appropriate. Furthermore, we did not test the scale's inter- and intra-observer reliability in this preliminary study.

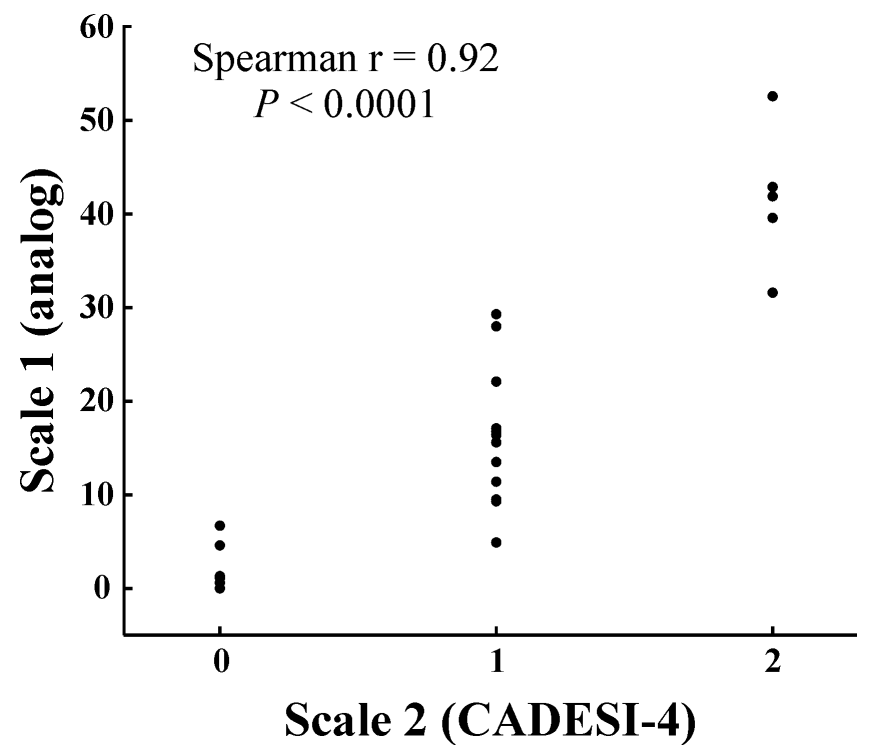

Figure 6. Correlation between the continuous/analog (Scale 1) and the step scale (CADESI-4, Scale 2).

With Skimager, we assessed the severity of erythema on 15 body sites (14 inguinal and one auricular measurement). We studied the relationship between the two scales and various EIs by linear regression analysis. Table 1 lists the results in terms of the coefficient of determination $\left(r^{2}\right)$. Both scales exhibited very similar results; however, $\mathrm{r}^{2}$ was slightly higher for the continuous scale. Averaging pixel values in two small squares (Fig. 5, SQ2) appeared the best sampling method. We speculate that sampling with one big square (SQ1) included extra skin components like hair and pigment, which affected the results. On the other hand, erythema distribution is typically uneven (patchy), making sampling with manually selected points (PT) challenging and biased.

Table 1. Coefficient of determination $\left(r^{2}\right)$ from the regression analysis between visual erythema scores based on the continuous (Scale 1, 0-100\%) and CADESI-4 scales (Scale 2, 0-3), and EIs (Eq. 1-4) relying on different sampling methods (PT - 60 points, SQ2 - two small squares, SQ1 - one big square). The best results are marked in bold.

\begin{tabular}{|c|c|c|c|c|c|c|c|c|c|c|c|c|}
\hline EI type & \multicolumn{3}{|c|}{ RG } & \multicolumn{3}{|c|}{ GR } & \multicolumn{3}{|c|}{ BRG } & \multicolumn{3}{|c|}{ BG } \\
\hline Sampling method & PT & SQ2 & SQ1 & $\mathrm{PT}$ & SQ2 & SQ1 & PT & SQ2 & SQ1 & $\mathrm{PT}$ & SQ2 & SQ1 \\
\hline Scale $1(0-100 \%)$ & 0.74 & 0.76 & 0.74 & 0.75 & 0.77 & 0.72 & 0.75 & 0.81 & 0.75 & 0.59 & 0.61 & 0.39 \\
\hline Scale $2(0-3)$ & 0.72 & 0.74 & 0.73 & 0.72 & 0.74 & 0.71 & 0.74 & 0.79 & 0.77 & 0.52 & 0.50 & 0.28 \\
\hline
\end{tabular}


The model based on $\mathrm{EI}_{\mathrm{BRG}}$ could predict visual erythema assessments the best, resulting in the coefficient of determination of 0.81 (Fig. 7). Except for one sample, there was no overlapping between CADESI-4 groups (Fig. 7(a)). Moreover, models based on $\mathrm{EI}_{\mathrm{RG}}$ and $\mathrm{EI}_{\mathrm{GR}}$ also offered a comparable prediction capability, resulting in the coefficients of determination of 0.76 and 0.77 , respectively. We should note that our results are not in agreement with the study of Saknite et al. ${ }^{8}$, where $\mathrm{EI}_{\mathrm{BG}}$ offered the best contrast between the normal and erythematous skin.
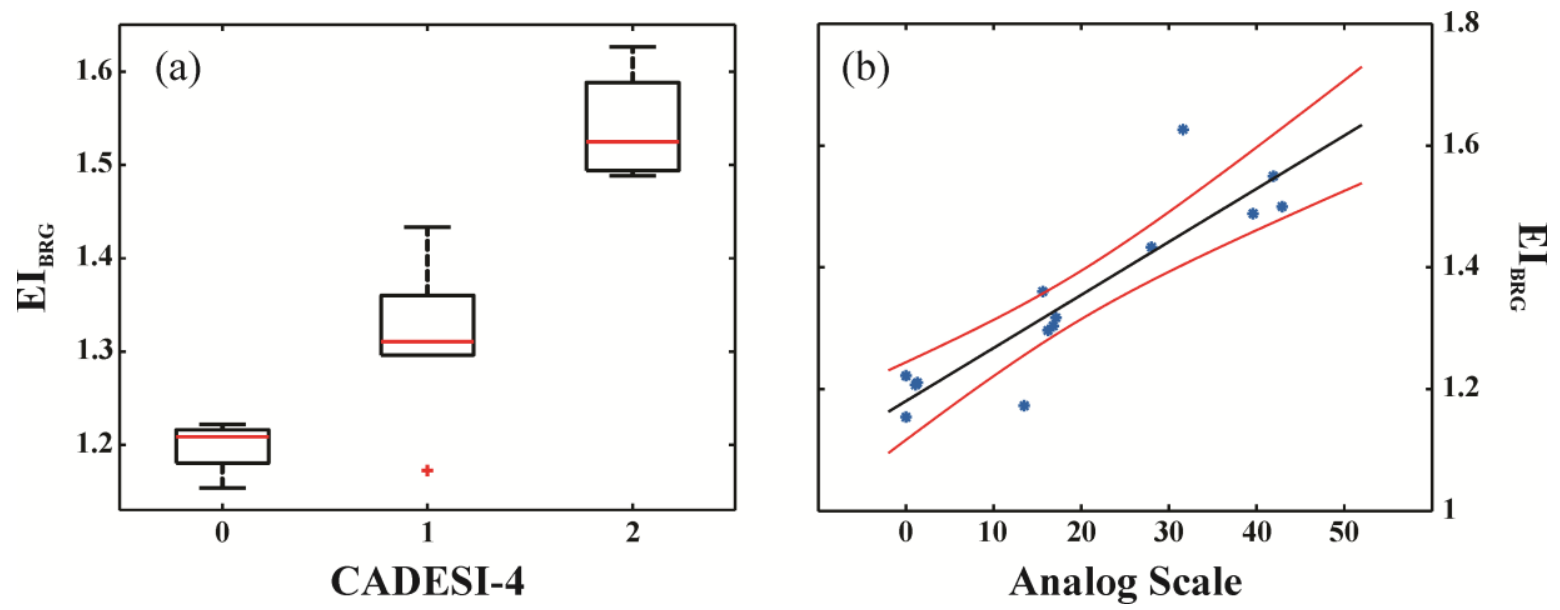

Figure 7. (a) EIBRG (Eq. 3), grouped according to the CADESI-4 scale (0-3). The red line represents the median. The bottom and top box edges indicate the 25th and 75th percentiles, respectively. Both whiskers present extreme data points. We mark outlier as a cross. (b) EIBRG and the corresponding values on the continuous scale (0-100\%) with a fitted linear regression line (black) and $95 \%$ confidence intervals (red lines).

\section{CONCLUSION}

In the study, we introduced a continuous (analog) scale, which could complement the existing discrete skin lesion scales for atopic dogs. However, the scale's inter- and intra-observer reliability should be tested before any clinical application. Secondly, the objective erythema estimation was done by calculating EIs based on multispectral skin images. Since the prediction models proved very reliable $\left(r^{2}=0.81\right)$, the objective estimations based on the optical devices like the Skimager could replace the manual erythema estimations for clinical or research work. However, the impact of other skin lesions (e.g., excoriation) or pigmentation on prediction models should be tested. Furthermore, the Skimager has some limitations in poorly accessible AD-related body sites like paws.

\section{ACKNOWLEDGMENTS}

The study was supported by the Marie Skłodowska-Curie Actions of the European Union (IF, DogSPEC, 745396).

\section{REFERENCES}

[1] Hensel, P., Santoro, D., Favrot, C., Hill, P. and Griffin, C., "Canine atopic dermatitis: detailed guidelines for diagnosis and allergen identification," BMC Vet. Res. 11(1), 196 (2015).

[2] Rybníček, J., Lau-Gillard, P. J., Harvey, R. and Hill, P. B., "Further validation of a pruritus severity scale for use in dogs," Vet. Dermatol. 20(2), 115-122 (2009).

[3] Olivry, T., Saridomichelakis, M., Nuttall, T., Bensignor, E., Griffin, C. E., Hill, P. B. and the International Committee on Allergic Diseases of Animals (ICADA)., "Validation of the Canine Atopic Dermatitis Extent and Severity Index (CADESI)-4, a simplified severity scale for assessing skin lesions of atopic dermatitis in dogs," Vet. Dermatol. 25(2), 77-85 (2014). 
[4] Plant, J. D., Gortel, K., Kovalik, M., Polissar, N. L. and Neradilek, M. B., "Development and validation of the Canine Atopic Dermatitis Lesion Index, a scale for the rapid scoring of lesion severity in canine atopic dermatitis," Vet. Dermatol. 23(6), 515-e103 (2012).

[5] Olivry, T., "Could we use the erythema grading of the CADESI4 as a simple instrument for future short-duration clinical trials of dogs with atopic dermatitis?," Vet. Dermatol. 30(1), 80-81 (2019).

[6] Olivry, T., Bensignor, E., Favrot, C., Griffin, C. E., Hill, P. B., Mueller, R. S., Plant, J. D., Williams, H. C. and for the International Committee of Allergic Diseases of Animals (ICADA)., "Development of a core outcome set for therapeutic clinical trials enrolling dogs with atopic dermatitis (COSCAD'18)," BMC Vet. Res. 14(1), 238 (2018).

[7] Hopkinson, D., Moradi Tuchayi, S., Alinia, H. and Feldman, S. R., "Assessment of rosacea severity: A review of evaluation methods used in clinical trials," J. Am. Acad. Dermatol. 73(1), 138-143.e4 (2015).

[8] Saknite, I., Zavorins, A., Jakovels, D., Spigulis, J. and Kisis, J., "Comparison of single-spot technique and RGB imaging for erythema index estimation," Physiol. Meas. 37(3), 333 (2016).

[9] Boysen, L., Sørensen, P., Larsen, M., Serup, J. and Kristensen, F., "Evaluation of skin erythema by use of chromametry and image analysis of digital photographs after intradermal administration of histamine in dogs," Am. J. Vet. Res. 63(4), 565-569 (2002).

[10] Spigulis, J., Garancis, V., Rubins, U., Zaharans, E., Zaharans, J. and Elste, L., “A device for multimodal imaging of skin," Proc.SPIE 8574, 85740J (2013).

[11] Spigulis, J., "Multispectral, fluorescent and photoplethysmographic imaging for remote skin assessment," Sensors 17(5) (2017).

[12] Spigulis, J., Rubins, U., Kviesis-Kipge, E. and Rubenis, O., "SkImager: a concept device for in-vivo skin assessment by multimodal imaging," Proc. Est. Acad. Sci. 63, 213 (2014). 